\title{
Comparison of Hemodynamic Performances Between Commercial Available Stents Design on Stenosed Femoropopliteal Artery
}

\author{
Che Mohammad Hafizal Muzammil Che Seman ${ }^{1}$, Nur Ayuni Marzuki ${ }^{1}$, Nofrizalidris Darlis ${ }^{1,}{ }^{*}$, Noraini \\ Marsi $^{1}$, Zuliazura Mohd Salleh ${ }^{1}$, Izuan Amin Ishak ${ }^{1}$, Ishkrizat Taib ${ }^{2}$, Safra Liyana Sukiman ${ }^{3}$ \\ 1 Advanced Technology Centre, Faculty of Engineering Technology, Universiti Tun Hussein Onn Malaysia (UTHM), Pagoh Education Hub, Pagoh, \\ Muar, Johor, Malaysia \\ 2 Faculty of Mechanical and Manufacturing Engineering, Universiti Tun Hussein Onn Malaysia (UTHM), Batu Pahat, Johor, Malaysia \\ 3 Centre for Language Studies, Universiti Tun Hussein Onn Malaysia (UTHM), Batu Pahat, Johor, Malaysia
}

\section{Article history:}

Received 20 May 2020

Received in revised form 19 July 2020

Accepted 24 July 2020

Available online 30 July 2020

\section{Keywords:}

Femoropopliteal artery; Commercials stent; Computational Fluid Dynamics (CFD)
Peripheral arteries disease (PAD) such as atherosclerosis is a disease where restriction of blood flow in the artery occurs when the size of arterial lumen narrows due to fat hardening and plaque deposition. PAD may cause claudication, critical limb ischemia as well as stroke. In recent years, endovascular approach of obstructive arterial disease such as femoropopliteal artery stenting is used as a treatment to overcome clotting. Stent is a small structure that provides scaffolding to hold arterial lumen open and allows blood flow through it. Therefore, many stents are developed to overcome the disease. Many researchers claimed that different shapes of stent geometry influenced the blood flow characteristics in the stented artery. This study aims to determine the hemodynamic performances of femoropopliteal artery that are stented with different commercial stents. Simulations are performed on stented femoropopliteal arteries to determine hemodynamic parameter of velocity distribution, pressure drops and wall shear stress (WSS) along the artery. From the results, the velocity and pressure distributions of the stented artery showed variant blood flow with the range of 0-0.19 $\mathrm{m} / \mathrm{s}$ and 14590-14730 $\mathrm{Pa}$ respectively. The pressure drops of the stented artery presents acceptable values below than $100 \mathrm{mmHg}$. The maximum of WSS is $2.52 \mathrm{~Pa}$ whilst the minimum of WSS is $1.61 \mathrm{~Pa}$. In summary, the Supera stent, Complete SE stent and Absolute Pro are chosen as superior hemodynamic performances when they represent stable low velocity, normal pressure including lowest pressure drops and lowest maximum wall shear stress as compared to other stents.

\footnotetext{
* Corresponding author.

E-mail address: nofrizal@uthm.edu.my (Nofrizalidris Darlis)
} 


\section{Introduction}

Femoropopliteal artery is one of the largest arteries located at the limb of lower extremity. The primary function of this arteryis to carry oxygenated blood from heart to be supplied to superficial tissues and thigh muscles. The restriction of blood flow in femoropopliteal artery will cause severe disease on patient known as peripheral arteries disease (PAD). The peripheral arteries disease such as thrombosis is caused by formation of plaque and fat hardening known as thrombus which occur in femoropopliteal artery. This disease will cause problems to the blood flow through the artery. The thrombosis in the femoropopliteal will cause claudication, in which cramping pain occurs in leg muscle due to the obstruction in the femoropopliteal artery. Other than that, Critical Limb Ischemia might also happen due narrowing artery [1]. Ischemia will cause severe tissues damage and loss of limb. Thus, coagulation of blood in the femoropopliteal artery needs to be treated at early stage in order to avoid fatal diseases.

Today, endovascular approach of obstructive arterial disease in the femoropopliteal artery is used as a treatment in stenosed artery. The treatment includes percutaneous transluminal angioplasty, stenting and atherectomy [2]. In recent years, stenting is used in many developed countries as a treatment to overcome clotting in the femoropopliteal artery. It was reported the range of technical success between $98-100 \%$ of the endovascular treatment of stenotic disease in femoropopliteal artery [3]. Numerous researchers studied various types of stent design such as bare metal nitinol stent, drug eluting stent and covered stent in order to improve stents in the femoropopliteal artery. In this study, simplified model geometry of common stenosed femoropopliteal artery will be determined for the stents design to be assigned to the artery for the purpose of evaluating hemodynamic differences using commercials Computational Fluid Dynamics (CFD) software. Computational fluid dynamics (CFD) approach is an excellent tool to examine micro features involved in the flow and it is widely used in flow analysis through stented arteries. Development of stenosis in artery is affected by hemodynamic parameters such as wall shear stress, velocity distributions and pressure drop. Hence, blood flow behavior in the artery is influenced by those parameters [4-5].

Over the past decades, endovascular technologies had been evolving rapidly, and innovative revascularization strategies such as stenting had been proposed for peripheral artery disease of femoropopliteal artery. However, it is still debatable about suitable design for the stenosed femoropopliteal artery. Hence, this study will investigate hemodynamic behaviours of velocity distribution, pressure drops and wall shear stress of different types of commercial stents design as better hemodynamic performances to benefit the treatment of the peripheral artery disease.

\section{Methodology}

\subsection{Simplified Geometry Model of Stented Femoropopliteal Artery}

In this study, part of femoropopliteal artery tree is simplified into a straight cylindrical model as shown in Figure 1. This femoropopliteal artery model is constructed based on the average size sought from previous study [6]. The artery is designed with the length of $120 \mathrm{~mm}$, having the outer and inner diameter of $6 \mathrm{~mm}$ and $5.8 \mathrm{~mm}$ respectively. Later, the model is stented with the length of $30 \mathrm{~mm}$. The stent is placed at $40 \mathrm{~mm}$ from inlet as the stenosis frequently identified there by Paisal et al., [7]. Simplified model is stimulated to study hemodynamic performance via different implantation of commercial available stents. 


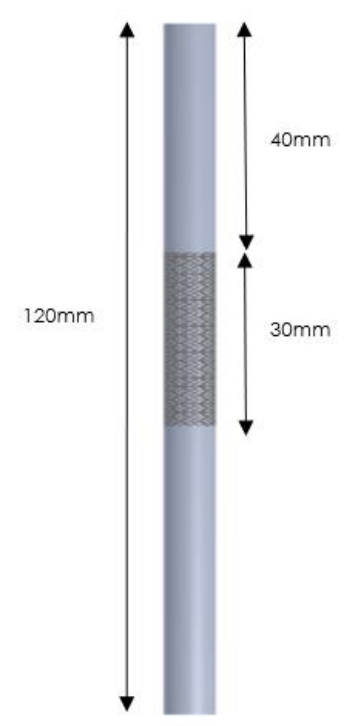

Fig. 1. Simplified stented femoropopliteal artery

\subsection{Stent Types and Designs}

Nine different commercial available stents design are simulated in this study. The configuration designs of selected stents are constructed based on the sizes available with the average length of 30 $\mathrm{mm}$. The stented femoropopliteal artery has the inner diameter of $5.8 \mathrm{~mm}$ and the outer diameter of $6 \mathrm{~mm}$ [8-12]. Each stents are expandable, thus it is implanted in the femoropopliteal artery of the inner diameter as highlighted in Figure 1. Detailed stent designs used in this study are illustrated in Figure 2.

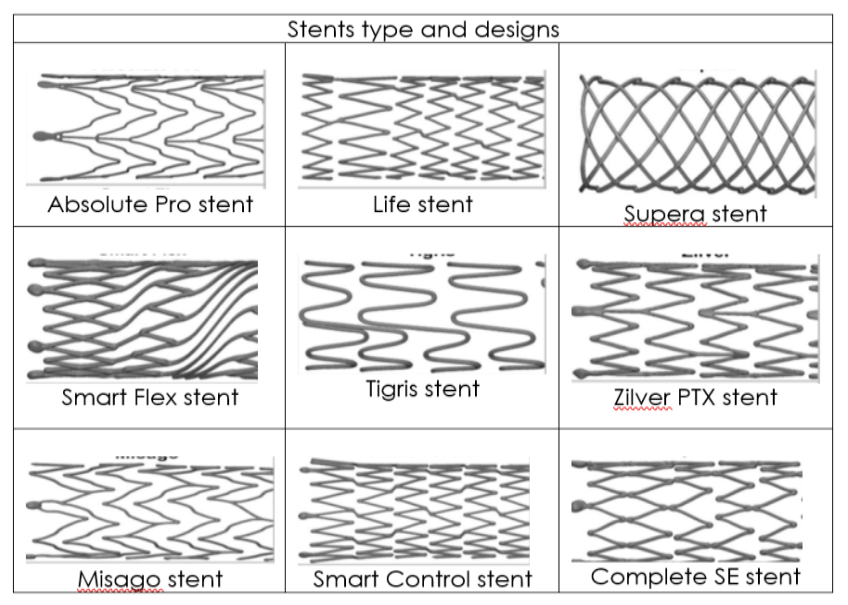

Fig. 2. Commercial available stents

\subsection{Computational Fluid Dynamics (CFD)}

A computational domain of simplified femoropopliteal artery model implanted with nine types of commercially available stents is drawn by using CAD software SOLIDWORKS 2018 (Dassault Systemes Solidworks Corporation, Waltham, MA).The models are transferred into ANSYS CFX 19.2 (Canonsburg, PA, USA) for discretization process of Navier-Stroke equation using finite volume method. Each models of computational domain is generated into tetrahedral mesh as highlighted in 
Figure 3. The tetrahedral mesh is considered due to the complexity of stent model and it is required to gain two points nodes between fluid domain and wall domain. To obtain the best result, finest meshing is applied near to stent strut configuration.

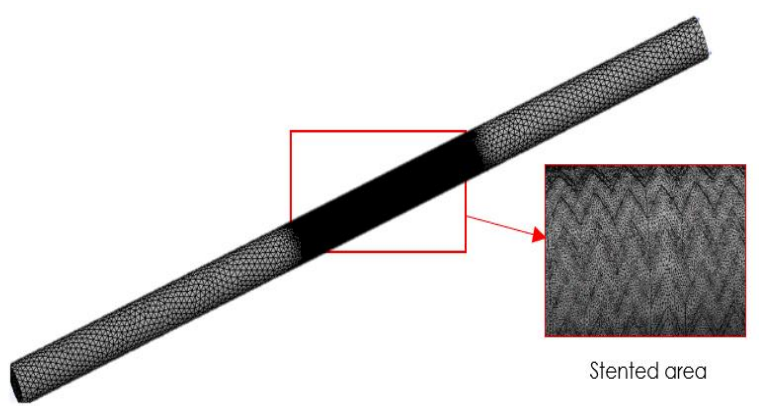

Fig. 3. Mesh of computational domain

The blood flow of femoropopliteal artery with stenting is assumed as incompressible and Newtonian fluid as the femoropopliteal artery is claimed to be one of the large artery [7]. As cited in numerous studies, Newtonian blood model is a suitable approximation blood model that can be used for numerical simulation pertinent blood flow in the artery. The density and viscosity of blood are $1050 \mathrm{~kg} / \mathrm{m} 3$ and 0.0035 Pa.s within the condition of the vessel to be considered as rigid and no slip between the vessel and stent [13-14]. Steady flow is assumed in the analysis of hemodynamic effect on all models of stent designs. The mean inlet velocity and outlet pressure placed as shown in Figure 4 are taken with the value of $0.10 \mathrm{~m} / \mathrm{s}$ and 14590 Pa respectively [7].

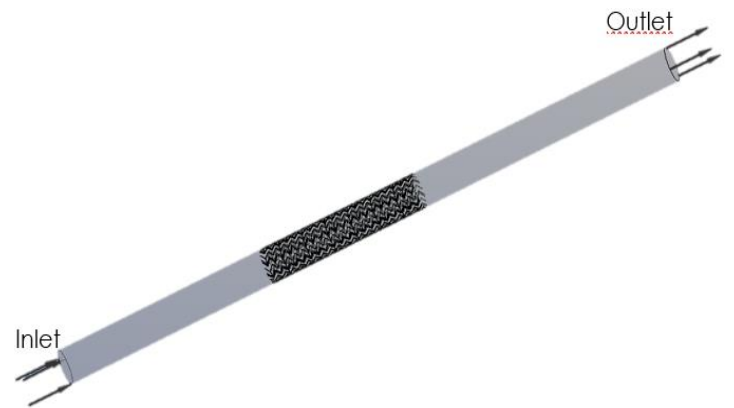

Fig. 4. Boundary condition of the stented femoropopliteal artery

The continuity and the Navier-Stokes equation are solved by computing velocity inlet and pressure outlet. Under the assumption of incompressibility, homogeneity and Newtonian blood, the Navier-Strokes equations are defined as illustrated in Eq. (1) and Eq. (2) [15-16].

$\rho \frac{\partial u}{\partial t}+\rho(u \cdot \nabla) u=-\nabla P+\mu \nabla^{2} u$

$\nabla \cdot u=0$

Where $u$ is the primary vector $u=[u, v, w]$, and $P$ is the pressure that vary in space $x, y, z$ and time, $t$.

Wall shear stress estimation is common analysis in predicting performance of medical devices towards vascular wall. Wall shear stress (WSS) can be defined as shown in Eq. (3).

$W S S_{\tau}=\frac{\partial v}{\partial r}$ 
Where $u$ is the fluid viscosity, $v$ is the velocity along the vessel axis, and $r$ is the perpendicular to and away from the wall [17].

\section{Results}

\subsection{Grid Independence Test}

Grid independence is a term used to describe improvement of results by using successive smaller cell sizes for calculations. The calculation should approach similar results as the mesh becomes finer, hence the term grid independence is derived. Figure 5 shows the velocity distributions of one stent model which is Complete SE stent with different number of nodes. This model is chosen to be the benchmark of the stent as this model comes with stable low velocity, normal pressure and lowest maximum wall shear stress among other stents. The size of the mesh is increased gradually from $300 \mathrm{k}$ to $400 \mathrm{k}$ nodes in order to observe significant changes of the velocity distribution. Based on the grid independence test, it can be seen that there are no significant changes of the velocity distribution with average relative error below 1 percent in velocity from the node of 350k to 400k nodes. Thus, subsequent simulations are set approximately greater or equal to $350 \mathrm{k}$ nodes. As this model achieves grid independence, the cell size should be successively smaller to minimize error due to discretization, and utilizing the same mesh setting on other models.

\subsection{Velocity Distribution}

The range of blood velocity distribution across the stented femoropopliteal artery is shown in Figure 6 with enlarged view of stented region. The results indicate that the highest maximum simulation goes to the Zilver PTX stent with the value of $0.457 \mathrm{~m} / \mathrm{s}$ whilst Tigris stent has the maximum velocity value of $0.390 \mathrm{~m} / \mathrm{s}$. Both stents exhibit slightly different maximum velocity as compared to other seven stent designs which depict the maximum velocity below than $0.19 \mathrm{~m} / \mathrm{s}$. Therefore, the maximum range velocity distribution of blood flow along the artery of $0.19 \mathrm{~m} / \mathrm{s}$ is chosen for this study. It can be seen that the flow region of all models near the strut of the stent decreases as compared to the core region. Slow movement can be seen clearly from the enlarged view of the stent region as shown in Figure 6. As expected, the velocity across the stent becomes slower as compared to the other regions. This happens because the design of the stent reduces the blood flow when the design and stent configuration play major role in determining suitable stent for the hemodynamic performance [13].

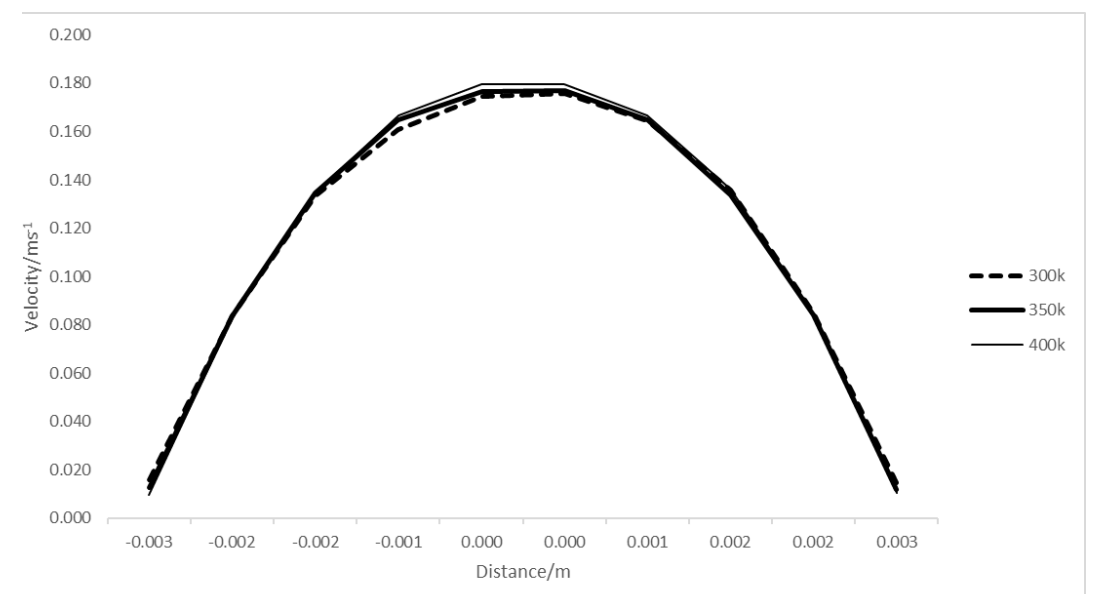

Fig. 5. Grid Independence test results of Complete SE stent with three difference node numbers 


\subsection{Pressure Drops}

Another factor that affects the hemodynamic performances is the pressure drops. Blood damage is likely to occur when there is large pressure drops in the artery. This is due to the high shear rate and outflow velocity from the inlet of the artery. Previous study has reported that hemolysis occurs when the blood flow pressure drops exceeding $100 \mathrm{mmHg}$ at flow rate of $4 \mathrm{~L} / \mathrm{min}$. Hence, the pressure drops below $100 \mathrm{mmHg}$ is used as a reference in this study [18]. Based on Figure 7, it is observed that each stented femoropopliteal artery has different pressure distribution. The inlet pressure for all stents are higher as compared to the outlet pressure. This is because when the velocity at the inlet issmaller, the pressure in each arteries will be larger. Two types of stents, namely Tigris stent and Misago stent exhibited the highest average of maximum pressure distribution of $14726 \mathrm{~Pa}$ amongother stented artery. However, there is no differences in regards with the average maximum pressure of other stented artery as the range is $14715-14722 \mathrm{~Pa}$. Moreover, there is also regions where higher pressure is produced on several stents configuration such as Life stent, Smart Flex stent, Tigris stent, Misago stent and Smart Control stent where red spots appear in Figure 7. This is possibly due to the effect of different strut configuration of the stents where irregular shape traps more pressure, thus increase resistance of the stents towards the blood flow and pressure distribution [7]. As expected, the highest pressure drops goes to the same stent which shows the maximum average of pressure as mentioned above. The pressure drops for the Tigris stent and the Misago stent are $1.02 \mathrm{mmHg}$ whilist the pressure drops of other stents depict Absolute Pro $=0.983$ $\mathrm{mmHg}$, Life stent $=1.005 \mathrm{mmHg}$, Supera stent $=0.938 \mathrm{mmHg}$, Smart Flex $=0.953 \mathrm{mmHg}$, Zilver PTX stent $=0.975 \mathrm{mmHg}$, Smart Control $=0.990 \mathrm{mmHg}$ and complete $\mathrm{SE}=0.938 \mathrm{mmHg}$.

In summary, all stented arteries exhibit pressure drops in which do not exceed beyond 100 $\mathrm{mmHg}$, suitable for clinical use as it can prevents hemolysis. Whilst the Supera stent and the Complete SE stent present the lowest pressure drops as compared to other stents. This findings are parallel with previous study that annotated lowest pressure drops can avoid hemolysis. However, selecting the best stent is needed as a precaution to determine hemodynamic performance.

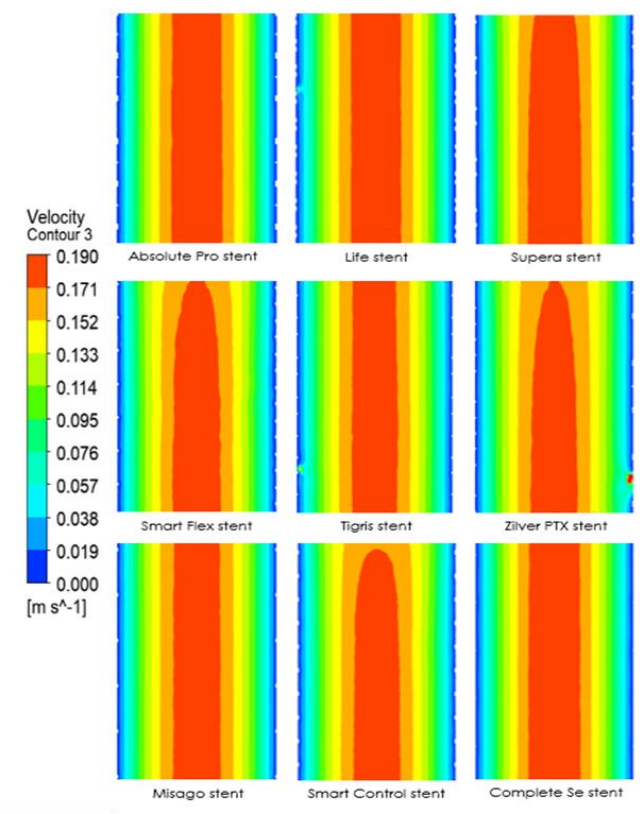

Fig. 6. Velocity distribution on stented femoropopliteal artery

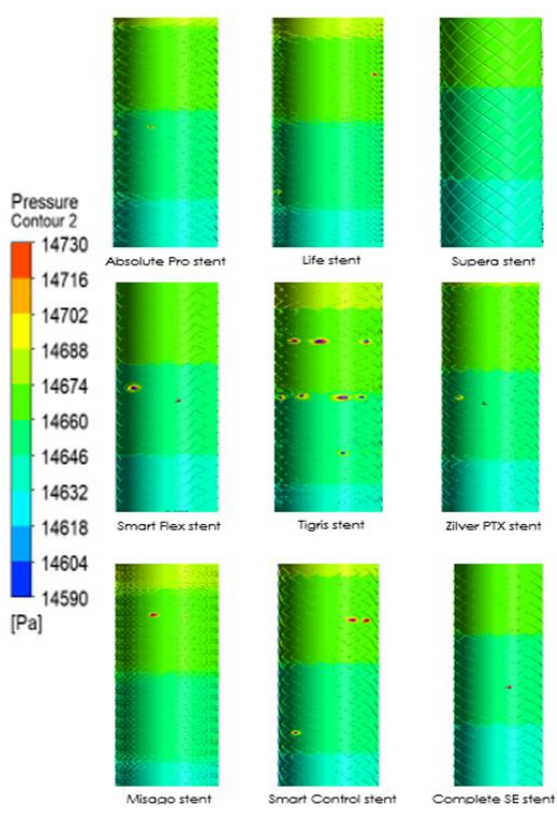

Fig. 7. Pressure distribution on stented femoropopliteal artery 


\subsection{Wall Shear Stress}

Wall shear stress plays an important role in determining blood flow to prevent thrombosis and atherosclerosis [14, 19-20]. Besides that, the design of the stent, or the stent configurations also influence the formation of the stenosis. Previous study has stated that the low shear stress has the value of lower than 4 dynes $/ \mathrm{cm}^{2}$ whereas the maximum shear stress should be greater than 15 dynes $/ \mathrm{cm}^{2}$ [21]. However, when the shear stress exceeds 70 dynes $/ \mathrm{cm}^{2}$, the formation of the thrombosis will likely to occur. Gogineni, further narrated in his study that the maximum shear stress in the bifurcated stented region of femoral artery is $12 \mathrm{~Pa}$ [22]. The simulation of the wall shear stress distributions along the stented artery with nine different stents design in this study simulates average arterial pressure.

Wall shear stress for nine stents design that have been stented in the femoropopliteal artery are observed as illustrated in Figure 8. Based from the observation, only two stents are beyond 70 dynes $/ \mathrm{cm}^{2}$ which corresponds to $7 \mathrm{~Pa}$. The stents mentioned belongs to the Supera stent and the Complete Se stent that show the lowest value of the wall shear stress of $3.832 \mathrm{~Pa}$ and $4.265 \mathrm{~Pa}$ respectively. Nevertheless, other stents present the maximum shear stress above $7 \mathrm{~Pa}$ which can lead to the formation of thrombosis. However, the range value of wall shear stress is set to 0-7 Pa in this study. This is because the percentage count of the area that has wall shear stress exceeding $7 \mathrm{~Pa}$ is smaller than $2 \%$. It is proven in the study as the percentage count of wall shear stress exceeding $7 \mathrm{~Pa}$ for the Absolute Pro stent $=0.11$ percent, the Life stent $=0.17$ percent, the Smart Flex stent $=0.27$ percent, the Tigris stent $=0.63$ percent, the Zilver PTX stent $=0.15$ percent, the Misago stent $=0.14$ percent, the Smart Control stent $=0.24$ percent. Therefore, the value of wall shear stress exceeding 7 Pa can be neglected.

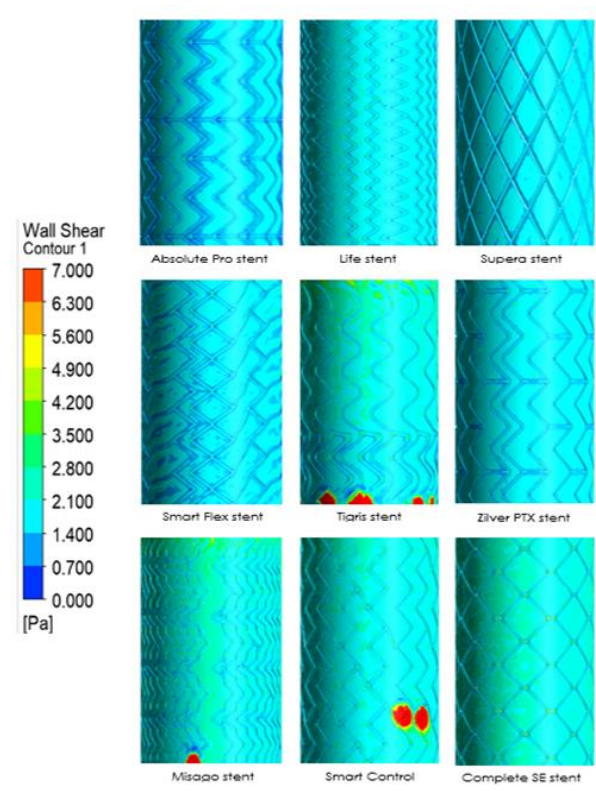

Fig. 8. Wall shear stress distribution on stented femoropopliteal artery

From the result, the maximum wall shear stress value can exceeds the percentage count of 2 percent. The Supera stent yields the lowest value of wall shear stress at $1.61 \mathrm{~Pa}$ with 2.4 percent of the count area. Other than that, the maximum wall shear stress value of $2.52 \mathrm{~Pa}$ belongs to the Life stent ( 2.2 percent), the Misago stent (2.2 percent) and the Smart Control stent ( 2 percent). The most suitable stent is the Supera stent as it exhibits the lowest wall shear stress as compared to other 
stents considering the parameter of wall shear stress. This is because hemodynamic parameter of wall shear stress distribution is influenced by the design and configurations of the stent. Paisal reported in his study that the design will have wall shear stress near to zero when the blood flow is stagnant due to the high wall shear stress. As a result, chances for the formation of atherosclerosis to happen in this condition is high. Therefore, blood flow should not be stagnant in the artery [7].

\section{Conclusions}

In conclusion, the comparison of hemodynamic performances between available commercial stents on stenosed femoropopliteal artery has been investigated in the present study. Computational fluid dynamic (CFD) analysis is performed on the stented femoropopliteal artery by using ANSYS software to analyse hemodynamic performances of velocity distribution, pressure drops and wall shear stress. Simulation is performed on stenosed artery with different available stent designs. Variant blood flow in all stented arteries are found in this study especially in velocity and pressure distributions along the stented artery. The findings also indicated that the maximum wall shear stress of all models of stented artery are acceptable as the percentages count areas of wall shear stress and pressure exceed 7 dynes $/ \mathrm{cm}^{2}$ are less than 2 percent. The Supera stent, the Complete SE stent and the Absolute Pro are chosen as superior hemodynamic performances when they represent stable low velocity, normal pressure including lowest pressure drops and lowest maximum wall shear stress as compared to other stents. Thus, essential shapes, configurations and design of the stents should be acknowledged in order to lower the risk of atherosclerosis formation due to the growth of thrombosis.

\section{Acknowledgement}

The authors would like to thank Ministry of Education Malaysia for supporting this research under Fundamental Research Grant Scheme Grant No. FRGS/1/2018/TK03/UTHM/03/7 and partially sponsored by Universiti Tun Hussein Onn Malaysia.

\section{References}

[1] M. Abu-fadel, A. Klein, E. J. Armstrong, H. Jeon-slaughter, E. S. Brilakis, and D. L. Bhatt. "Peripheral Vascular Disease." (2016): 1-11.

[2] Mehta, Manish, Yi Zhou, Philip SK Paty, Medhi Teymouri, Kamran Jafree, Humayun Bakhtawar, Jeffrey Hnath, and Paul Feustel. "Percutaneous common femoral artery interventions using angioplasty, atherectomy, and stenting." Journal of vascular surgery 64, no. 2 (2016): 369-379.

https://doi.org/10.1016/i.jvs.2016.03.418

[3] Diamantopoulos, Athanasios, and Konstantinos Katsanos. "Treating femoropopliteal disease: established and emerging technologies." In Seminars in interventional radiology, vol. 31, no. 04, pp. 345-352. Thieme Medical Publishers, 2014. https://doi.org/10.1055/s-0034-1393971

[4] Khader, Shah Mohammed Abdul, Adi Azriff, Cherian Johny, Raghuvir Pai, Mohammad Zuber, Kamarul Arifin Ahmad, and Zanuldin Ahmad. "Haemodynamics Behaviour in Normal and Stenosed Renal Artery using Computational Fluid Dynamics." Journal of Advanced Research in Fluid Mechanics and Thermal Sciences 51, no. 1 (2018): 80-90.

[5] Basri, Adi Azriff, Shah Mohammed Abdul Khader, Cherian Johny, Raghuvir Pai, Muhammad Zuber, Kamarul Arifin Ahmad, and Zanuldin Ahmad. "Numerical Study of Haemodynamics Behaviour in Normal and Single Stenosed Renal Artery using Fluid-Structure Interaction." Journal of Advanced Research in Fluid Mechanics and Thermal Sciences 51, no. 1 (2018): 91-98.

[6] Mohammad, Shafiullah, and Pradip Majumdar. "Computational Fluid Dynamics Analysis of Blood Flow Through Stented Arteries." In ASME International Mechanical Engineering Congress and Exposition, vol. 56215, p. V03AT03A047. American Society of Mechanical Engineers, 2013. https://doi.org/10.1115/IMECE2013-62407

[7] Paisal, Muhammad Sufyan Amir, Syed Fadhil Syed Adnan, Ishkrizat Taib, Al Emran Ismail, Mohammad Kamil 
Abdullah, Normayati Nordin, Suzairin Md Seri, and Nofrizalidris Darlis. "Flow Characteristics Near to Stent Strut Configurations on Femoropopliteal Artery." In IOP Conference Series: Materials Science and Engineering, vol. 226, no. 1, p. 012147. 2017.

https://doi.org/10.1088/1757-899X/226/1/012147

[8] Brescia, Alexander A., Brian M. Wickers, Juan Carlos Correa, Mathew R. Smeds, and Donald L. Jacobs. "Stenting of femoropopliteal lesions using interwoven nitinol stents." Journal of Vascular Surgery 61, no. 6 (2015): 1472-1478. https://doi.org/10.1016/i.jvs.2015.01.030

[9] Wu, Ridong, Zilun Li, Mian Wang, Guangqi Chang, Chen Yao, and Shenming Wang. "Paclitaxel-coated versus uncoated balloon angioplasty for femoropopliteal artery in-stent restenosis." International Journal of Surgery 42 (2017): 72-82. https://doi.org/10.1016/j.ijsu.2017.04.057

[10] Nikanorov, Alexander, Martin Schillinger, Hugh Zhao, Erich Minar, and Lewis B. Schwartz. "Assessment of selfexpanding nitinol stent deformation after chronic implantation into the femoropopliteal arteries." Eurolntervention 9, no. 6 (2013): 730-737.

https://doi.org/10.4244/EIJV9I6A117

[11] Duda, Stephan H., Marc Bosiers, Johannes Lammer, Dierk Scheinert, Thomas Zeller, Vincent Oliva, Alexander Tielbeek et al. "Drug-eluting and bare nitinol stents for the treatment of atherosclerotic lesions in the superficial femoral artery: long-term results from the SIROCCO trial." Journal of Endovascular Therapy 13, no. 6 (2006): 701710. https://doi.org/10.1583/05-1704.1

[12] Lucatelli, Pierleone, Marco Cini, Giulio Tommasino, Antonio Benvenuti, Giulia Guaccio, Stefano Bascetta, Eugenio Neri, and Carmelo Ricci. "Use of the Gore Tigris Vascular Stent in advanced femoropopliteal peripheral arterial disease." Journal of Vascular and Interventional Radiology 29, no. 5 (2018): 614-622.

https://doi.org/10.1016/i.jvir.2017.12.006

[13] Jiang, Yongfei, Jun Zhang, and Wanhua Zhao. "Influence of strut cross-section of stents on local hemodynamics in stented arteries." Chinese Journal of Mechanical Engineering 29, no. 3 (2016): 624-632. https://doi.org/10.3901/CJME.2016.0125.013

[14] Wensing, Peter JW, Louis Meiss, Willem PTM Mali, and Berend Hillen. "Early atherosclerotic lesions spiraling through the femoral artery." Arteriosclerosis, thrombosis, and vascular biology 18, no. 10 (1998): 1554-1558. https://doi.org/10.1161/01.ATV.18.10.1554

[15] Campo-Deaño, Laura, Mónica SN Oliveira, and Fernando T. Pinho. "A review of computational hemodynamics in middle cerebral aneurysms and rheological models for blood flow." Applied Mechanics Reviews 67, no. 3 (2015). https://doi.org/10.1115/1.4028946

[16] Thomas, S., Shirly Empaling, Nofrizalidris Darlis, Kahar Osman, Jeswant Dillon, Ishkrizat Taib, and Ahmad Zahran Md Khudzari. "Computational modelling of flow and tip variations of aortic cannulae in cardiopulmonary bypass procedure." In IOP Conf Ser Mater Sci Eng, vol. 243, p. 012021. 2017. https://doi.org/10.1088/1757-899X/243/1/012021

[17] Shaaban, Akram M., and André J. Duerinckx. "Wall shear stress and early atherosclerosis: a review." American Journal of Roentgenology 174, no. 6 (2000): 1657-1665.

https://doi.org/10.2214/ajr.174.6.1741657

[18] Brodman, Richard, Howard Siegel, Martin Lesser, and Robert Frater. "A comparison of flow gradients across disposable arterial perfusion cannulas." The Annals of thoracic surgery 39, no. 3 (1985): 225-233. https://doi.org/10.1016/S0003-4975(10)62584-2

[19] Kim, Young-Ho, Jong-Eun Kim, Yasushi Ito, Alan M. Shih, Brigitta Brott, and Andreas Anayiotos. "Hemodynamic analysis of a compliant femoral artery bifurcation model using a fluid structure interaction framework." Annals of biomedical engineering 36, no. 11 (2008): 1753. https://doi.org/10.1007/s10439-008-9558-0

[20] Smedby, Örjan, Sven Nilsson, and Lott Bergstrand. "Development of femoral atherosclerosis in relation to flow disturbances." Journal of biomechanics 29, no. 4 (1996): 543-547.

https://doi.org/10.1016/0021-9290(95)00070-4

[21] A. M. Malek and S. L. Alper, "Hemodynaamics Shear Stress and Its Role in Atherosclerosis," vol. 282, no. 21, pp. 2035-2042, 2 Malek, Adel M., Seth L. Alper, and Seigo Izumo. "Hemodynamic shear stress and its role in atherosclerosis." Jama 282, no. 21 (1999): 2035-2042.013. https://doi.org/10.1001/jama.282.21.2035

[22] A. Gogineni, "Flow Characteristics and Wall Shear Stresses in Bifurcated Peripheral Arteries," Wichita State University, 20 Gogineni, Aneesha. "Flow characteristics and wall shear stresses in bifurcated peripheral arteries." PhD diss., Wichita State University, 2015. 